\title{
Estimation of Combining Ability and Gene Action by Using Line X Tester Procedure in Bread Wheat (Triticumastivum., L).
}

Nesma S. hussain , M. El. M. El-Badawy,A.A.A. El Hosary, S.A.S Mehasen

Agron.Dept., Fac. of Agric. Moshtohor, Benha Univ.

Corresponding Author: ahmed.alhossary@ fagr.bu.edu.eg

\begin{abstract}
The present research was conducted to study the combining ability and gene action for morphological, yield and its components traits in F1 crosses of wheat using line $\mathrm{x}$ tester procedure. Experiment was conducted incloudingthirty genotypes consisting of ten lines, L1,L2, L3, L4, L5, L6, L7, L8, L9, L10and three testers namely; Sids 12 (T1), Gemmeiza 11 (T2) and Line 137 ((T3).Thirtycrosses and their parents were evaluated under two irrigation treatments in randomized complete block design with three replications at experimental Research Farm, Faculty of Agriculture Benha Univ. during the winter successive growing seasons 2017/18 and 2018/19. The obtained results could be summarized as follows; highly significant differences were found among irrigation treatment, genotypes and its partitioning lines, testers and line $\mathrm{x}$ testers for all the studied traits. Also, interaction between irrigation treatment and mention source of variance were significant for most traits under study. Genetic analysis revealed that the importance of both additive and non-additive components in the inheritance of all traits. The non-additive was more important for most studied traits. The best general combining ability was L8 for earliness, plant height, No. of spikes /plant and grain yield. The crosses T1 x L3, T2 x L1, T2 x L2, T2 x L4, T3 x L4 and T3 x L6 showed highly significant positive Sij effects (desirable) for biological yield/ plant and grain yield/ plant.
\end{abstract}

Key words: Wheat, combining ability, drought stress, GCA and SCA.

\section{Introduction}

Bread wheat (Triticumaestivum L.) is a major food crop in the world. In Egypt, it is used as a stable food grain for urban, rural and bedewing societies and as a major source of straw for animal feeding. However, geometrical increase in the Egyptian population has been a challenge for agricultural scientists. To improve yield potential of wheat there is requirement to have knowledge regarding the nature of combining ability of available parents to be used in the hybridization programme and also about the nature of gene action involved in the expression of economically important quantitative as well as qualitative traits Hassan et al. (2007), Al Saadoon (2017),AL Sadoonet al. (2019) and Al-Tamimi et al (2020). For the development of genetically superior high yielding varieties, identification of superior parents is an important pre-requisite. Earlier research review revealed that both general and specific combining ability were involved for yield and yield components, Chaudhryet al.(1992), Al Saadoon et al. (2017), EL-Hosary et al. (2019) and Al-Tamimi et al (2020). For effective improvement in yield of wheat, one can use combining ability analysis to test the performance of selected parents in different cross combinations and can characterise the nature and magnitude of gene effects in the expression of various yield contributing traits.

Keeping the above in view, the present line $x$ tester analysis was planned to estimate general and specific combining ability effects to identify better parents as well as high cross combinations for further improvement in wheat.

\section{Material and Methods}

The present study was carried out at Faculty of Agriculture at moshtohor, Egypt during 2017/18and 2018/19 seasons, Egypt. In 2017/18 season, line x tester mating design was performed through 10lines in addition to three testers to produce the hybrid seeds of 30 crosses. Parents of the beforementioned genotypes are listed in Table 1.

In $2018 / 19$, the 13 parents along with the $30 \mathrm{~F}_{1}$ 'S were grown in randomized complete block design with three replications. The sowing date was on 4th Dec. 2018. Two adjacent experiments were conducted. The first experiment was irrigated only once after planting irrigation and the second one was normally irrigated five irrigations. Each genotype was grown in 2 rows of $3 \mathrm{~m}$ length with inter row and intra row spacing of $30 \mathrm{~cm}$ and $10 \mathrm{~cm}$ respectively. To raise a good crop stand all the recommended cultural practices were followed. The recommended cultural practices were applied at the proper time. Data were recorded on a sample of 10 plants/replication in each genotypes whereas for days to $50 \%$ flowering (day),plant height $(\mathrm{cm})$, number of spikes per plant, number of grains per spike, 1000-grain weight $(\mathrm{g})$ and grain yield per plant (g). Data for the characters depicting significant difference were further analyzed for line $\mathrm{x}$ tester according to Singh and Chaudhry (1979). 
Table 1. The name and pedigrees of the parental genotypes.

\begin{tabular}{|c|c|c|c|}
\hline NO. & Genotype & Pedigree & Origin \\
\hline $\mathrm{L}_{1}$ & M101 & MILAN \S71101 \\
OAPYMex & CIMMYT \\
\hline $\mathrm{L}_{2}$ & M102 & MILAN \S7102\\
OAPYMex & CIMMYT \\
\hline $\mathrm{L}_{3}$ & M103 & MILAN \S7103 \OAPYMex & CIMMYT \\
\hline $\mathrm{L}_{4}$ & M104 & MILAN \S7104 \ OAPYMex & CIMMYT \\
\hline $\mathrm{L}_{5}$ & M105 & MILAN \S7105 \ OAPYMex & CIMMYT \\
\hline $\mathrm{L}_{6}$ & M106 & MILAN \S7106 \ OAPYMex & CIMMYT \\
\hline $\mathrm{L}_{7}$ & M107 & MILAN \S7107\\
OAPYMex & CIMMYT \\
\hline $\mathrm{L}_{8}$ & M108 & MILAN \S7108 \OAPYMex & CIMMYT \\
\hline $\mathrm{L}_{9}$ & M109 & MILAN \S7109 \OAPYMex & CIMMYT \\
\hline $\mathrm{L}_{10}$ & M110 & MILAN \S7110 \ OAPYMex & CIMMYT \\
\hline $\mathrm{T} 1$ & Sids 12 & $\begin{array}{l}\text { BUC//7C/ALD/5/MAYA74/ON//1160.147/3/BB/GLL } \\
\text { /4/CHAT"S"/6/MAYA/VUL//CMH74A.630/ } \\
\text { 4*SXSD7096-4SD-1SD-1SD 0SD }\end{array}$ & Egypt \\
\hline $\mathrm{T} 2$ & GEMMEIZA 11 & $\begin{array}{l}\text { BOW"S"/KVS"S"//7C/SERI82/3/GIZA168/SAKHA61- GM- } \\
\text { 7892-2GM-1GM2GM-1GM-0GM }\end{array}$ & Egypt \\
\hline $\mathrm{T} 3$ & M L 1137 & MILAN \S7137\\
OAPYMex & Egypt \\
\hline
\end{tabular}

\section{Results and Discussion}

\section{Analysis of variance}

The analysis of variance of ordinary and line $\mathrm{x}$ testers mating design for all the studied traits are presented in Table (2).

Analysis of variance revealed highly significant for crossesand their partitions; lines, testers and line $\mathrm{x}$ testers for all the studied traits except thousand grain weight for line, tester and line $\mathrm{x}$ tester in both and across environments and tester for plant high in drought environment, indicating the wide diversity among the genotypes, which is considered adequate for further biometrical assessment.These results are in agreement with those of Abd El-Aty (2002), Abd El-Atyand Katta (2002), Nouret al. (2011), Kumar et al. (2015) and AlTamimi et al (2020).

\section{Genetic components}

Knowledge of gene action helps in the selection of parents for use in the hybridization programs, also in the choice of appropriate breeding procedure for the genetic improvement quantitative characters.

The estimates of genetic parameters were calculated, for all the studied traits as presented in table (2). The results showed that the non-additive genetic variance were larger than the additive genetic variance $\sigma^{2} \mathrm{SCA}$ for all the studied traits, suggesting greater importance of non-additive genetic variance in the inheritance of these traits. The GCA varince were lower than SCA varince in terms of all traits evaluated in the research in contrast to Titan $\boldsymbol{e t}$ al. (2012). They used 6 wheat lines and seven testers and they tested 42 F1 combinations for two seasons. Also, Sharma et al. (2006) stated that $\sigma^{2}$ gca variance was of greater importance than $\sigma^{2}$ sca for some traits. The difference in the results reported by researchers may be attributed to differences of parental materials used hybridization and to genotype $\times$ environments. The ratio $\sigma^{2} \mathrm{GCA} / \sigma^{2} \mathrm{SCA}$ varies depending on the allele frequencies between parental populations (Reifet al., 2007; Longinet al., 2013). The lines selected from different gene pools had favorable $\sigma^{2} \mathrm{GCA} / \sigma^{2} \mathrm{SCA}$ ratio because of their high GCA effects (Labateet al.,1997).In this study, low ratios of $\mathrm{O}^{2} \mathrm{GCA} / \mathrm{O}^{2} \mathrm{SCA}, \mathrm{O}^{2} \mathrm{~A} / \mathrm{O}^{2} \mathrm{D}$ and These results showed that $\sigma^{2} \mathrm{GCA} / \sigma^{2} \mathrm{SCA}$ portion was lower than one and $\left(\mathrm{O}^{2} \mathrm{~A} / \mathrm{O}^{2} \mathrm{D}\right)$ portion, which is an indicator of dominancy degree, lower than one (Table 2). Hence, it can be seen that non-additive genetic effects are controlling the inheritance of studied traits. Fellahiet al. (2013) and EL-Hosary and Abdelwahed (2015) reported the importance of non-additive gene action for the plant height, number of fertile tillers, thousand kernel weight and kernel yield. They recommended that selection of superior plants should be postponed to later generations due to preponderance of non-additive type of gene actions for all studied traits. Similar results of predominance of non-additive gene action for all studied traits have been reported by Vermaet al. (2007) for wheat. The efficiency of the selection is related with the size of narrow sense heritability in the segregating populations. The heritability degrees were found very low for the traits studied in the research (Table 2). This situation showed that the additive variance is very low in this population and the selection must be applied in the further generations. 
This findings proved that in the present study, both non-aditive and additive components are important expression of the studied traits. Similar results were previously reported by Khalifaet al (1998), Abd El-
Aty (2000),Abd El-Aty and Katta (2002), ELHosary and Abdelwahed (2015) and Al-Tamimi et al (2020).

Table 2. Analysis of variance for various agronomic traits in line $\mathrm{x}$ tester analysis in wheat.

\begin{tabular}{|c|c|c|c|c|c|c|c|c|}
\hline S.O.V. & df & $\begin{array}{c}\text { Days to } \\
\text { heading } \\
\text { (days) }\end{array}$ & $\begin{array}{l}\text { Plant } \\
\text { height } \\
(\mathrm{cm})\end{array}$ & $\begin{array}{l}\text { No. of } \\
\text { spikes } \\
\text { /plant }\end{array}$ & $\begin{array}{l}\text { No. of } \\
\text { grains } \\
\text { /spike }\end{array}$ & $\begin{array}{c}\text { 1000- } \\
\text { Grain } \\
\text { weigh } \\
\text { t } \\
(\mathrm{g})\end{array}$ & $\begin{array}{c}\text { Biological } \\
\text { yield/ } \\
\text { plant } \\
\text { (g) }\end{array}$ & $\begin{array}{c}\text { Grain } \\
\text { yield/plan } \\
\text { t } \\
(\mathrm{g})\end{array}$ \\
\hline \multicolumn{9}{|c|}{ Normal irrigation treatment } \\
\hline Rep & 2 & $41.88 * *$ & 0.93 & 8.63 & 1.88 & 0.08 & 2.63 & 0.74 \\
\hline Crosses (c) & 29 & $11.86 * *$ & $76.95 * *$ & $113.21 * *$ & $246.90 * *$ & 1.16 & $7994.06 * *$ & $368.48 * *$ \\
\hline Line $(\mathbf{L})$ & 9 & $12.62 * *$ & $47.54 * *$ & $49.99 * *$ & $191.17 * *$ & 0.80 & $5250.96 * *$ & $426.54 * *$ \\
\hline Tester (T) & 2 & $2.41 *$ & $\begin{array}{c}191.10 * \\
*\end{array}$ & $47.56 * *$ & $\begin{array}{c}1056.14 * \\
*\end{array}$ & 1.54 & $4291.30 * *$ & $369.38 * *$ \\
\hline $\mathbf{L} \mathbf{x} \mathbf{T}$ & 18 & $12.52 * *$ & $78.98 * *$ & $152.12 * *$ & $184.85 * *$ & 1.30 & $9777.03 * *$ & $339.35 * *$ \\
\hline Error & 58 & 5.81 & 11.35 & 2.76 & 1.23 & 0.78 & 7.96 & 3.92 \\
\hline$\delta_{\mathrm{GCA}}^{2}$ & & -0.01 & -0.04 & -0.73 & 1.16 & 0.00 & -33.34 & 0.54 \\
\hline$\delta^{2} \mathrm{SCA}$ & & 2.24 & 22.54 & 49.79 & 61.20 & 0.17 & 3256.36 & 111.81 \\
\hline \multicolumn{9}{|c|}{ Droughttreatment } \\
\hline $\operatorname{Rep}$ & 2 & 7.14 & 2.01 & 19.87 & 0.83 & 1.03 & 0.58 & 4.04 \\
\hline Crosses (c ) & 29 & $17.84 * *$ & $78.07 * *$ & $228.04 * *$ & $\begin{array}{c}2877.67 * \\
*\end{array}$ & 1.40 & $2543.49 * *$ & $268.79 * *$ \\
\hline Line (L) & 9 & $37.09 * *$ & $\begin{array}{c}124.73 * \\
*\end{array}$ & $196.21 * *$ & $\begin{array}{c}5514.80 * \\
*\end{array}$ & 1.02 & $1708.57 * *$ & $134.23 * *$ \\
\hline Tester (T) & 2 & $8.01 * *$ & 0.57 & $263.81 * *$ & $\begin{array}{c}1196.94 * \\
*\end{array}$ & 1.03 & $9712.34 * *$ & $123.94 * *$ \\
\hline $\mathbf{L} \times \mathbf{T}$ & 18 & $9.31 * *$ & $63.35^{* *}$ & $239.98 * *$ & $\begin{array}{c}1745.86^{*} \\
*\end{array}$ & 1.63 & $2164.41 * *$ & $352.16 * *$ \\
\hline Error & 58 & 4.18 & 6.34 & 1.76 & 1.25 & 1.00 & 10.28 & 3.59 \\
\hline$\delta 2 G C A$ & & 0.16 & 0.28 & 0.00 & 21.16 & 0.00 & 7.09 & -1.56 \\
\hline ס2SCA & & 1.71 & 19.00 & 1.06 & 581.54 & 0.21 & 718.04 & 116.19 \\
\hline \multicolumn{9}{|c|}{ Combined across irrigation treatment } \\
\hline Irrigation & & & $576.38^{*}$ & & $1328.45^{*}$ & & $75727.02 *$ & \\
\hline (I) & 1 & $26.45 * *$ & $*$ & $32688.11 * *$ & $*$ & $6.42 * *$ & $*$ & $11.55^{* *}$ \\
\hline $\operatorname{Rep} / \mathbf{I}$ & 4 & $24.51 * *$ & 1.47 & $14.25^{* *}$ & 1.36 & 0.56 & 1.61 & 2.39 \\
\hline Crosses (c) & 29 & $6.67 * *$ & $44.98 * *$ & $107.02 * *$ & $\begin{array}{c}1030.96 * \\
*\end{array}$ & 0.93 & $3687.41 * *$ & $239.83 * *$ \\
\hline Line (L) & 10 & $7.96 * *$ & $42.82 * *$ & $59.66 * *$ & $\begin{array}{c}1925.14 * \\
*\end{array}$ & 0.85 & $2379.14 * *$ & $180.62 * *$ \\
\hline Tester (T) & 2 & 1.16 & $\begin{array}{c}106.12 * \\
*\end{array}$ & $43.78 * *$ & $487.82 * *$ & 2.07 & $755.49 * *$ & $357.54 * *$ \\
\hline $\mathbf{L} \times \mathbf{T}$ & 18 & $6.42 * *$ & $41.69 * *$ & $135.22 * *$ & $622.66 * *$ & 0.89 & $4550.97 * *$ & $261.03 * *$ \\
\hline CxI & 29 & $12.90 * *$ & $57.20 * *$ & $117.89 * *$ & $\begin{array}{c}1028.42 * \\
*\end{array}$ & 0.76 & $3257.79 * *$ & $180.18 * *$ \\
\hline LxI & 9 & $24.00 * *$ & $67.93 * *$ & $98.61 * *$ & $\begin{array}{c}1742.98 * \\
*\end{array}$ & 0.32 & $2094.84 * *$ & $179.87 * *$ \\
\hline TxI & 2 & $9.27 * *$ & $85.55 * *$ & $267.60 * *$ & $\begin{array}{c}1765.27 * \\
*\end{array}$ & 0.51 & $\begin{array}{c}13248.16^{*} \\
*\end{array}$ & $135.78 * *$ \\
\hline LxTxI & 18 & $7.61 * *$ & $49.80 * *$ & $116.84 * *$ & $618.50 * *$ & 0.99 & $3125.66^{* *}$ & $183.51 * *$ \\
\hline Error & 11 & & & & & & & \\
\hline Err & 6 & 3.29 & 5.83 & 1.49 & 0.82 & 0.59 & 6.01 & 2.47 \\
\hline$\delta^{2} \mathrm{GCA}$ & & -0.20 & 0.11 & -2.77 & -10.22 & 0.02 & -139.44 & 0.62 \\
\hline$\delta^{2} \mathrm{SCA}$ & & -0.20 & -1.35 & 3.06 & 0.69 & -0.02 & 237.55 & 12.92 \\
\hline$\delta^{2}{ }_{G C A x I}$ & & 0.24 & 0.60 & -2.58 & 8.96 & 0.00 & -147.96 & 1.27 \\
\hline$\delta^{2} S C A x I$ & & 1.44 & 14.66 & 38.45 & 205.90 & 0.14 & 1039.88 & 60.35 \\
\hline
\end{tabular}

*and $* *$ significant at 0.05 and 0.01 respectively 


\section{Mean performance of genotypes}

Mean performance values of the $F_{1}$ crosses for all the studied traits are presented in Table (3). The crosses $\mathrm{T}_{1} \times \mathrm{L}_{2}, \mathrm{~T}_{1} \times \mathrm{L}_{8}, \mathrm{~T}_{1} \times \mathrm{L}_{9}, \mathrm{~T}_{2} \times \mathrm{L}_{5}, \mathrm{~T}_{2} \times \mathrm{L}_{7}, \mathrm{~T}_{2} \times$ $\mathrm{L}_{8}$ and $\mathrm{T}_{3} \times \mathrm{L}_{9}$ were the earliest flowering date. As the regard to plant height, four of the $F_{1}$ top crosses $T_{1} X$

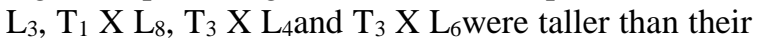
parental means. The cross T2xL8 had the highest values for No.of spikes/plant. The parental combination T1xL5 had higherNo. of grains/spike.
The cross T3xL1 were superior in 1000-grain weight. Concerning the grain yield/plant, the cross T2xL1 were higher in the grain yield/plant.The crosses were higher in the grain yield/plant, where the heaviest cross for biological yield/ plant was detected by the cross T1xL3. These results were coincident with these obtained by Khalifaet al.(1998), Abd El-Aty and Katta (2002), Nouret al.(2011), Kumar et al. (2015) and Rajput and Kandalkaret al.(2018).

Table 3. Mean performance of hybrids (line $\mathrm{x}$ tester) for all studied traits.

\begin{tabular}{|c|c|c|c|c|c|c|c|}
\hline Cross & Days to heading (Days) & $\begin{array}{c}\text { Plant } \\
\text { height } \\
(\mathrm{cm})\end{array}$ & $\begin{array}{l}\text { No. of } \\
\text { spikes } \\
\text { /plant }\end{array}$ & $\begin{array}{l}\text { No. of } \\
\text { grain } \\
\text { /spike }\end{array}$ & $\begin{array}{c}\text { 1000- } \\
\text { Grain } \\
\text { weight } \\
\text { (g) }\end{array}$ & $\begin{array}{l}\text { biological yield/ plant } \\
(\mathrm{g})\end{array}$ & $\begin{array}{c}\text { grain } \\
\text { yield/ } \\
\text { plant } \\
\text { (g) }\end{array}$ \\
\hline T1 X L1 & 102.33 & 102.83 & 25.01 & 62.17 & 46.67 & 237.33 & 50.83 \\
\hline T1XL2 & 99.83 & 108.17 & 17.46 & 64.17 & 51.67 & 292.67 & 46.33 \\
\hline T1XL3 & 104.83 & 111.83 & 25.06 & 51.50 & 56.67 & 368.17 & 68.83 \\
\hline T1X L4 & 102.67 & 110.00 & 18.78 & 68.17 & 50.00 & 231.33 & 42.13 \\
\hline T1XL5 & 101.17 & 104.33 & 19.96 & 137.67 & 53.33 & 252.83 & 61.83 \\
\hline T1XL6 & 103.00 & 101.67 & 29.29 & 60.33 & 46.67 & 243.50 & 54.17 \\
\hline T1XL7 & 103.67 & 104.17 & 22.01 & 58.67 & 45.00 & 301.00 & 44.10 \\
\hline T1XL8 & 100.83 & 112.50 & 18.20 & 60.33 & 46.67 & 266.33 & 54.17 \\
\hline T1X L9 & 99.00 & 103.83 & 33.36 & 66.50 & 56.67 & 292.00 & 61.83 \\
\hline T1XL10 & 101.17 & 104.33 & 21.90 & 56.67 & 51.67 & 278.50 & 55.67 \\
\hline T2 X L1 & 102.00 & 104.50 & 31.03 & 53.67 & 48.33 & 306.33 & 75.00 \\
\hline T2XL2 & 103.33 & 104.83 & 27.85 & 67.50 & 48.33 & 296.17 & 56.33 \\
\hline T2XL3 & 102.83 & 103.67 & 21.44 & 67.00 & 55.00 & 262.83 & 56.17 \\
\hline T2X L4 & 101.17 & 106.33 & 26.58 & 54.33 & 40.00 & 278.67 & 54.83 \\
\hline T2XL5 & 100.83 & 104.17 & 22.06 & 84.33 & 50.00 & 270.33 & 56.83 \\
\hline T2XL6 & 102.33 & 100.17 & 17.12 & 60.33 & 41.67 & 245.50 & 42.00 \\
\hline T2XL7 & 100.33 & 108.00 & 20.28 & 67.17 & 40.00 & 248.50 & 51.50 \\
\hline T2XL8 & 100.83 & 108.00 & 39.44 & 51.50 & 50.00 & 286.50 & 53.50 \\
\hline T2X L9 & 101.83 & 104.50 & 16.50 & 70.33 & 55.00 & 241.17 & 41.50 \\
\hline T2XL10 & 102.33 & 103.00 & 20.14 & 59.33 & 48.33 & 257.00 & 56.53 \\
\hline T3 X L1 & 103.33 & 107.83 & 23.10 & 57.67 & 58.33 & 322.33 & 59.00 \\
\hline T3XL2 & 104.00 & 109.15 & 23.25 & 70.17 & 55.00 & 252.83 & 65.17 \\
\hline T3XL3 & 101.33 & 109.33 & 17.49 & 63.33 & 48.33 & 256.17 & 53.83 \\
\hline T3X L4 & 101.17 & 110.83 & 22.33 & 53.33 & 53.33 & 310.33 & 62.50 \\
\hline T3XL5 & 101.67 & 103.00 & 20.65 & 80.33 & 46.67 & 241.67 & 54.17 \\
\hline T3XL6 & 103.50 & 113.50 & 28.89 & 51.17 & 55.00 & 263.83 & 55.83 \\
\hline T3XL7 & 101.67 & 106.00 & 19.86 & 73.83 & 50.00 & 267.67 & 56.00 \\
\hline T3XL8 & 101.50 & 103.83 & 23.60 & 73.50 & 45.00 & 263.00 & 62.33 \\
\hline T3X L9 & 100.67 & 103.33 & 22.49 & 49.67 & 46.67 & 248.67 & 51.17 \\
\hline T3XL10 & 101.67 & 106.67 & 24.04 & 65.17 & 53.33 & 307.50 & 64.17 \\
\hline LSD 5\% & 2.05 & 2.73 & 1.38 & 1.02 & 46.67 & 2.77 & 1.78 \\
\hline LSD $1 \%$ & 2.70 & 3.59 & 1.81 & 1.34 & 51.67 & 3.65 & 2.34 \\
\hline
\end{tabular}

Combining ability

General combining ability effects (GCA ( $(\hat{g} i))$.

The estimate of general combining ability for parents (lines and testers) are presented in Table (4).
The result illustrated that: the tester 1 was a good combiner for No of grains/ spike and biological yield/ plant. T2 showed good combiner for plant height. T3 was a good combiner for plant height, 1000-grain weight and grain yield/ plant. 
Table 4. Estimates of general combining ability effects of parents for studied traits.

\begin{tabular}{|c|c|c|c|c|c|c|c|}
\hline Parent/Line & $\begin{array}{c}\text { Days to } \\
\text { heading } \\
\text { (days) }\end{array}$ & $\begin{array}{c}\text { Plant } \\
\text { height } \\
\text { (cm) }\end{array}$ & $\begin{array}{l}\text { No. of } \\
\text { spikes } \\
\text { /plant }\end{array}$ & $\begin{array}{l}\text { No. of } \\
\text { grains } \\
\text { /spike }\end{array}$ & $\begin{array}{c}\text { 1000- } \\
\text { Grain } \\
\text { weight } \\
\text { (g) }\end{array}$ & $\begin{array}{c}\text { biological } \\
\text { yield/ } \\
\text { plant } \\
\text { (g) }\end{array}$ & $\begin{array}{c}\text { Grain } \\
\text { yield/ } \\
\text { plant } \\
(\mathrm{g})\end{array}$ \\
\hline \multicolumn{8}{|l|}{ Tester } \\
\hline 1 & -0.04 & 0.22 & -0.20 & $3.29 * *$ & 0.07 & $3.34 * *$ & $-1.62 * *$ \\
\hline 2 & -0.11 & $-1.43 * *$ & $0.94 * *$ & $-1.78 * *$ & $-0.21 * *$ & $-3.72 * *$ & $-1.19 * *$ \\
\hline 3 & 0.16 & $1.20 * *$ & $-0.73 * *$ & $-1.51 * *$ & $0.14 *$ & 0.38 & $2.81 * *$ \\
\hline L.S.D. (gi) $5 \%$ & 0.32 & 0.43 & 0.22 & 0.16 & 0.14 & 0.44 & 0.28 \\
\hline L.S.D. (gi) $1 \%$ & 0.43 & 0.57 & 0.29 & 0.21 & 0.18 & 0.58 & 0.37 \\
\hline L.S.D. (gi-gj) $5 \%$ & 0.53 & 0.71 & 0.36 & 0.26 & 0.22 & 0.72 & 0.46 \\
\hline L.S.D. (gi-gj) $1 \%$ & 0.70 & 0.93 & 0.47 & 0.35 & 0.29 & 0.94 & 0.60 \\
\hline \multicolumn{8}{|l|}{ Line } \\
\hline 1 & 0.66 & -1.09 & $3.07 * *$ & $-7.49 * *$ & 0.13 & $15.64 * *$ & $6.00 * *$ \\
\hline 2 & 0.49 & $1.24 *$ & -0.46 & $1.95 * *$ & 0.19 & $7.53 * *$ & 0.34 \\
\hline 3 & $1.11^{* *}$ & $2.13 * *$ & $-1.98 * *$ & $-4.72 * *$ & $0.36^{*}$ & $22.70 * *$ & $4.00 * *$ \\
\hline 4 & -0.23 & $2.91 * *$ & $-0.74 *$ & $-6.72 * *$ & -0.20 & 0.42 & $-2.45 * *$ \\
\hline 5 & -0.67 & $-2.31 * *$ & $-2.42 * *$ & $35.45^{* *}$ & 0.02 & $-18.08 * *$ & $2.00 * *$ \\
\hline 6 & $1.05^{*}$ & -1.03 & $1.79 * *$ & $-8.05 * *$ & -0.20 & $-22.08 * *$ & $-4.94 * *$ \\
\hline 7 & -0.01 & -0.09 & $-2.59 * *$ & $1.23 * *$ & $-0.48 * *$ & -0.63 & $-5.08 * *$ \\
\hline 8 & $-0.84 *$ & $1.97 * *$ & $3.77 * *$ & $-3.55 * *$ & -0.26 & -1.08 & $1.06 * *$ \\
\hline 9 & $-1.39 * *$ & $-2.26 * *$ & $0.81 * *$ & $-3.16 * *$ & 0.30 & $-12.41 * *$ & $-4.11 * *$ \\
\hline 10 & -0.17 & $-1.48 * *$ & $-1.28 * *$ & $-4.94 * *$ & 0.13 & $7.98 * *$ & $3.18 * *$ \\
\hline L.S.D. (gi) $5 \%$ & 0.84 & 1.12 & 0.56 & 0.42 & 0.35 & 1.13 & 0.73 \\
\hline L.S.D. (gi) $1 \%$ & 1.10 & 1.47 & 0.74 & 0.55 & 0.46 & 1.49 & 0.96 \\
\hline L.S.D. (gi-gj) $5 \%$ & 1.19 & 1.58 & 0.80 & 0.59 & 0.50 & 1.60 & 1.03 \\
\hline L.S.D. (gi-gj) $1 \%$ & 1.56 & 2.07 & 1.05 & 0.78 & 0.66 & 2.10 & 1.35 \\
\hline
\end{tabular}

*and ** significant at 0.05 and 0.01 respectively

Also, the results revealed that twoparental lines; $\mathrm{L}_{8}$, and $\mathrm{L}_{9}$ showed significant negative (ĝi) effects (desirable) for days to heading is essentially a prerequisite in breeding program of a crop.

Regarding to plant height, dwarf plants are more lodging resistant while tall plants are preferred for straw purpose thus preference depends upon the breeding objective. Therefore, the parental lines $\mathrm{L}_{2}$, $\mathrm{L}_{3}, \mathrm{~L}_{4}$, and $\mathrm{L}_{8}$ can be considered as good general combiner for tallness as they showed highly significant positive (ĝi) effects, while, L5, L9 and L10 exhibited good combiner for dwarfness as they showed highly significant negative GCA (ĝi) .

For No.of spikes/plant, the parental lines $\mathrm{L}_{1}, \mathrm{~L}_{6}$, $\mathrm{L}_{8}$ and $\mathrm{L}_{9}$ showed highly significant positive (ĝi) effects.

With respect to No. of grains/spike parental line $\mathrm{L}_{2}$, showed highly significant positive GCA effects, these parents considered as good combiner for this trait.No. of grains/spike is an important yield contributing trait.

For 1000-grains weight, the parental line; $\mathrm{L}_{3}$ showed highly significant positive (ĝi) effects. 1000 grain weight is an important direct selection criterion for the selection of grain yield, thus significant positive GCA values considered as good general combining ability effects.
Regarding biological yield/ plant and grain yield/plant; three parental lines $\mathrm{L}_{1}$, $\mathrm{L}_{3}$ and $\mathrm{L}_{10}$ exhibited highly significant positive (ĝi) effects. These results are in agreement with the earlier studies carried out by Abd El-Aty and Katta (2002), Akbar et al. (2009), Abdel Nouret al (2011), Attiaet al. (2014), Kumar et al (2015), Abro et al (2016) and Tabassum and parasad (2017).

\section{Specific combining ability}

The results of specific combining ability effects of top crosses for all the studied traits are presented in Table (5).

Three crosses, $\mathrm{T}_{1} \times \mathrm{L}_{2}, \mathrm{~T}_{1} \times \mathrm{L}_{9}$, and $\mathrm{T}_{3} \times \mathrm{L}_{3}$ showed significant negative (desirable) Sij effectsfor days to heading, which indicated that one or more of these combinations could be helpful for selecting early maturity wheat lines.

For plant height, five crosses $\mathrm{T}_{1} \times \mathrm{L}_{3}, \mathrm{~T}_{1} \times \mathrm{L}_{8}, \mathrm{~T}_{2}$ $\times \mathrm{L}_{7}, \mathrm{~T}_{2} \times \mathrm{L}_{9}$ and $\mathrm{T}_{3} \times \mathrm{L}_{6}$ showed significant positive Sij effects (tall plant), it could be a good combiner for straw production.

For No.of spikes/plant, thetop crosses; $\mathrm{T}_{1} \times \mathrm{L}_{3}$, $\mathrm{T}_{1} \times \mathrm{L}_{6}, \mathrm{~T}_{1} \times \mathrm{L}_{7}, \mathrm{~T}_{1} \times \mathrm{L}_{9}, \mathrm{~T}_{2} \times \mathrm{L}_{1}, \mathrm{~T}_{2} \times \mathrm{L}_{2}, \mathrm{~T}_{2} \times \mathrm{L}_{4}$, $\mathrm{T}_{2} \times \mathrm{L}_{8}, \quad \mathrm{~T}_{3} \times \mathrm{L}_{2}, \quad \mathrm{~T}_{3} \times \mathrm{L}_{6}$ and $\mathrm{T}_{3} \times \mathrm{L}_{10}$ showed significant positive (desirable) SCA (Sij). These crosses can be used for increasing No. of tillers/plant. 
Regarding No.of grains/spike, the crosses; $\mathrm{T}_{1} \mathrm{x}$ $\mathrm{L}_{1}, \mathrm{~T}_{1} \times \mathrm{L}_{4}, \mathrm{~T}_{1} \times \mathrm{L}_{5}, \mathrm{~T}_{1} \times \mathrm{L}_{9}, \mathrm{~T}_{2} \times \mathrm{L}_{2}, \mathrm{~T}_{2} \times \mathrm{L}_{3}, \mathrm{~T}_{2} \times$ $\mathrm{L}_{6}, \mathrm{~T}_{2} \times \mathrm{L}_{7}, \mathrm{~T}_{2} \times \mathrm{L}_{9}, \mathrm{~T}_{3} \times \mathrm{L}_{1}, \mathrm{~T}_{3} \times \mathrm{L}_{2}, \mathrm{~T}_{3} \times \mathrm{L}_{3}$, $\mathrm{T}_{3} \times \mathrm{L}_{7}, \mathrm{~T}_{3} \times \mathrm{L}_{8}$ and $\mathrm{T}_{3} \times \mathrm{L}_{10}$ exhibited significant positive Sij effects.

Thirteen and fourteen crosses exhibited positive Sij effects for biological yield/ plant and grain yield/plant, respectively. However, the crosses $\mathrm{T}_{1} \mathrm{x}$ $\mathrm{L}_{3}, \quad \mathrm{~T}_{2} \times \mathrm{L}_{1}, \quad \mathrm{~T}_{2} \times \mathrm{L}_{2}, \mathrm{~T}_{2} \times \mathrm{L}_{4}, \mathrm{~T}_{3} \times \mathrm{L}_{4}$ and $\mathrm{T}_{3} \times$ $\mathrm{L}_{6}$ showed highly significant positive $\mathrm{Sij}$ effects (desirable) for the mention traits, they consider the best combiner for both traits.

Table 5. Estimates of specific combining ability effects of parents for studied traits.

\begin{tabular}{|c|c|c|c|c|c|c|c|}
\hline cross & $\begin{array}{l}\text { Days to } \\
\text { heading }\end{array}$ & $\begin{array}{l}\text { Plant } \\
\text { height }\end{array}$ & $\begin{array}{l}\text { No. of } \\
\text { spikes } \\
\text { /plant }\end{array}$ & $\begin{array}{l}\text { No. of } \\
\text { grains } \\
\text { /spike }\end{array}$ & $\begin{array}{c}\text { 1000- } \\
\text { Grain } \\
\text { weight }\end{array}$ & $\begin{array}{c}\text { biological } \\
\text { yield/ } \\
\text { plant }\end{array}$ & $\begin{array}{l}\text { Grain } \\
\text { yield/ } \\
\text { plant }\end{array}$ \\
\hline T1 X L1 & -0.18 & $-2.45^{*}$ & $-1.17 *$ & $1.04 * *$ & -0.52 & $-54.68 * *$ & $-9.16 * *$ \\
\hline T1XL2 & $-2.51 * *$ & 0.56 & $-5.19 * *$ & $-6.40 * *$ & -0.07 & $8.77 * *$ & $-7.99 * *$ \\
\hline T1XL3 & $1.88^{*}$ & $3.33 * *$ & $3.93 * *$ & $-12.40 * *$ & 0.26 & $69.10 * *$ & $10.84 * *$ \\
\hline T1X L4 & 1.04 & 0.72 & $-3.58 * *$ & $6.27 * *$ & 0.15 & $-45.46^{* *}$ & $-9.40 * *$ \\
\hline T1XL5 & -0.01 & 0.28 & -0.73 & $33.60 * *$ & 0.26 & $-5.46 * *$ & $5.84 * *$ \\
\hline T1XL6 & 0.10 & $-3.67 *$ & $4.39 * *$ & -0.23 & -0.18 & $-10.79 * *$ & $5.12 * *$ \\
\hline T1XL7 & $1.82 *$ & $-2.11 *$ & $1.50 * *$ & $-11.18 * *$ & -0.07 & $25.27 * *$ & $-4.81 * *$ \\
\hline T1XL8 & -0.18 & $4.17 * *$ & $-8.67 * *$ & $-4.73 * *$ & -0.13 & $-8.96 * *$ & -0.88 \\
\hline T1X L9 & $-1.46^{*}$ & -0.28 & $9.45^{* *}$ & $1.04 * *$ & 0.32 & $28.04 * *$ & $11.95 * *$ \\
\hline T1XL10 & -0.51 & -0.56 & 0.08 & $-7.01 * *$ & -0.02 & $-5.84 * *$ & $-1.50 * *$ \\
\hline $\mathrm{T} 2 \mathrm{X}$ L1 & -0.44 & 0.87 & $3.71 * *$ & $-2.39 * *$ & -0.07 & $21.39 * *$ & $14.58 * *$ \\
\hline T2XL2 & 1.06 & -1.12 & $4.06 * *$ & $2.00 * *$ & -0.12 & $19.33 * *$ & $1.58^{*}$ \\
\hline T2XL3 & -0.06 & $-3.18 *$ & -0.83 & $8.17 * *$ & 0.38 & $-29.17 * *$ & $-2.26 * *$ \\
\hline T2X L4 & -0.39 & -1.30 & $3.08 * *$ & $-2.50 * *$ & -0.57 & $8.94 * *$ & $2.87 * *$ \\
\hline T2XL5 & -0.28 & 1.76 & 0.23 & $-14.67 * *$ & 0.21 & $19.11 * *$ & 0.41 \\
\hline T2XL6 & -0.50 & $-3.52 * *$ & $-8.92 * *$ & $4.83 * *$ & -0.40 & -1.72 & $-7.48 * *$ \\
\hline T2XL7 & -1.44 & $3.37 * *$ & $-1.37 * *$ & $2.39 * *$ & -0.29 & $-20.17 * *$ & $2.16^{* *}$ \\
\hline T2XL8 & -0.11 & 1.32 & $11.42 * *$ & $-8.50 * *$ & 0.49 & $18.28 * *$ & $-1.98 * *$ \\
\hline T2X L9 & 1.44 & $2.04 *$ & $-8.56^{* *}$ & $9.94 * *$ & 0.43 & $-15.72 * *$ & $-8.81 * *$ \\
\hline T2XL10 & 0.72 & -0.24 & $-2.83 * *$ & 0.72 & -0.07 & $-20.28 * *$ & -1.07 \\
\hline T3 X L1 & 0.62 & 1.57 & $-2.54 * *$ & $1.34 * *$ & 0.58 & $33.29 * *$ & $-5.42 * *$ \\
\hline T3XL2 & $1.46^{*}$ & 0.56 & $1.13 *$ & $4.40 * *$ & 0.19 & $-28.10 * *$ & $6.41 * *$ \\
\hline T3XL3 & $-1.82 *$ & -0.15 & $-3.11 * *$ & $4.23 * *$ & $-0.64 *$ & $-39.93 * *$ & $-8.59 * *$ \\
\hline T3X L4 & -0.66 & 0.57 & 0.50 & $-3.77 * *$ & 0.42 & $36.51 * *$ & $6.54 * *$ \\
\hline T3XL5 & 0.29 & $-2.04 *$ & 0.50 & $-18.93 * *$ & -0.47 & $-13.66 * *$ & $-6.25 * *$ \\
\hline T3XL6 & 0.40 & $7.18 * *$ & $4.53 * *$ & $-4.60 * *$ & 0.58 & $12.51 * *$ & $2.36 * *$ \\
\hline T3XL7 & -0.38 & -1.26 & -0.13 & $8.79 * *$ & 0.36 & $-5.10 * *$ & $2.66^{* *}$ \\
\hline T3XL8 & 0.29 & $-5.48 * *$ & $-2.75 * *$ & $13.23 * *$ & -0.36 & $-9.32 * *$ & $2.86^{* *}$ \\
\hline T3X L9 & 0.01 & -1.76 & -0.89 & $-10.99 * *$ & $-0.75^{*}$ & $-12.32 * *$ & $-3.14 * *$ \\
\hline T3XL10 & -0.21 & 0.80 & $2.75 * *$ & $6.29 * *$ & 0.08 & $26.12 * *$ & $2.57 * *$ \\
\hline L.S.D. (Sij) 5\% & 1.45 & 1.93 & 0.98 & 0.72 & 0.61 & 1.96 & 1.26 \\
\hline L.S.D. (Sij) $1 \%$ & 1.91 & 2.54 & 1.28 & 0.95 & 0.80 & 2.58 & 1.65 \\
\hline L.S.D. (Sij-Skl) 5\% & 2.05 & 2.73 & 1.38 & 1.02 & 0.87 & 2.77 & 1.78 \\
\hline L.S.D. (Sij-Skl) $1 \%$ & 2.70 & 3.59 & 1.81 & 1.34 & 1.14 & 3.65 & 2.34 \\
\hline
\end{tabular}

*and ** significant refer to the level of significant at 0.05 and 0.01 respectively

\section{References}

Abd El-Aty, M.S. and Y.S. Katta (2002) Genetic analysis and heterosis grain yield and related traits in bread wheat (Triticumaestivum L.). J. Agric. Res. Tanta Univ., 28 (2) 2002.

Abd El-Aty,M.S.M. (2000) Estimates of heterosis and combining ability in diallel wheat crosses (Triticumaestivum L.). J. Agric. Tanta Univ. 26(3)486-498.

AbdelNour, N. A. R.; H. S.A.EL-Fateh and A.K.Mostafa (2011). Line $x$ Tester analysis for yield and its traits in bread wheat. Egypt. J. Agric. Res., 89 (3):979-992.

Abro, S.A.; A.W. Baloch; M. Baloch; G. A.Baloch; T. A. Baloch; A. A. Soomro; Q. Jogi and M. Ali (2016). Line $x$ Tester analysis for estimating combining ability in $\mathrm{f} 1$ hybrids of bread wheat.Pure Appl. Biol., 5(3): 647-652.

Akbar, M.; A. Rehman; M.H. Chaudhry and M. Hussain (2009).Linextester analysis in bread wheat (TriticumaestivumL.). J Agri Res. 47(1): 411-420. 
Allard, R. W. (1960).Principles of Plant Breeding. John Willey and Sons, New York, London.

Al Saadoon A.W., A.A. EL Hosary, A. S. Sedhom, M.EL.M. EL-Badawy, A.A.A. Hosary (2017). Genetic analysis of diallel crosses in wheat under stress and normal irrigation treatments. Egypt. J. Plant Breed.21 (5): 279-292.

AL Sadoon A.W. , A.A. EL Hosary , S. A. Sedhom , M.EL.M. EL-Badawy and A.A.A. El Hosary (2018). Determination of Combining Ability and Genetic Diversity Using ISSR Markers to Evaluate the Genetic Variability in Wheat.Journal Tikrit Univ. For Agri. Sci. Vol. (18) special No. of The 7 th Scientific and 1st International Conference of Agricultural Researches, 10-11 April 2018. 67-80.

Al-TamimiO.A., J.M.A. Al-Jbori and A.A.A. ElHosary (2020) Genetic analysis of $F 1$ cross in wheat (Triticumasetivum L.). Plant Archives 20: 413-4137.

Attia, S.A.A, Abed N.R, El-Hameid and A.A. Haiba (2014).Heterosis, combining ability analysis of some bread wheat crosses and the genetic relationship among the included studied cultivars. J App Sci Res. 9(10): 6394-6403.

Chaudhry, M.A.; M.S. Akhtarand; M.T. Ahmad (1992).Combining ability analysis for flag leaf area, yield and its components in spring wheat.J. of Agri. Research, 30(1):17-23.

EL-Hosary A.A.A., El Saeed M. El-Gedwy and M.A. Abdel-Salam (2019)Utilization of ISSR marker and tolerance indices for selecting adapted wheat genotypes under water stress. Bioscience Research, 16(2): 1611-1625.

EL-Hosary A.A.A. and G.A.A.Abdelwahed (2015) Heterosis and combining ability in F1 and F2 generations of diallel cross in wheat. Egypt. J. Plant Breed. (19) (2): 355-373.

Fellahi, Z.E.A., A.Hannachi, H.Bouzerzour and A.Boutekrabt (2013).Linextester mating desing analysis for grain yield and yield related traits in bread wheat (Triticumaestivum L.). Int. J. of Agro.:9.

Hassan, G.; F. Mohammad; S.S. Afridi and I. Khalil (2007).Combining ability in the F1 generations and yield components in Wheat.Sarhad. J. Agric., 23(4): 937-942.

Khalifa, M.A.; A.A.Ismail; G.R. El-Nagar and I.A. (1998).Genetical studies of earliness and grain yield and its components of bread wheat. Assuit J. of Agric. Sci. vol. 29 No. 5, 59-69.
Kumar, A., Harshwardhan; A. kumar and B. Prasad (2015). Combining ability and gene interaction study for yield, its attributing traits and quality in common wheat. J. of Applied and Nat. Sci., 7(2): 927-934.

Labate, J.A.; K. R. Lamkey; M. Lee and W. L. Woodman.(1997). Molecular genetic diversity after reciprocal recurrent selection in BSSS and BSCB1 maize populations. Crop Sci. 37: 416423.

Longin, C.F.;M. Gowda; J. Mühleisen; E. Ebmeyer; E. Kazman; R. Schachschneider; J. Schacht; M. Kirchhoff; Y. Zhao and J. C. Reif. (2013). Hybrid wheat: quantitative genetic parameters and consequences for the design of breeding programs. Theor. Appl. Genet. 126(11):2791-801

Nour, A.; A.R. Nadya; H.S.A. El-Fateh and A.K. Mostafa (2011).Line x Tester analysis for yield and its traits in bread wheat.Egypt J.Agric. Res., 89 (3):979-990.

Rajput, R.S. and V.S. Kandalkar (2018).Combining ability and heterosis for grain yield and its attributing traits in bread wheat (Triticumaestivum L.).J. of Phar.And Phyt. 7(2):113-119.

Reif, J.C.; F. Gumpert; S. Fischer and A. E. Melchinger (2007).Impact of genetic divergence and dominance variance in hybrid populations. Genetics 176: 1931-1934.

Sharma, H.C.; M.K. Dhillan and B. V. S. Reddy (2006).Expression of resistance to Atherigonasoccatain F1 hybrids involving shoot fly-resistant and susceptible cytoplasmic malesterile and restorer lines of sorghum. Plant breeding 125: 473-477.

Singh, R. K. and B. D. Chaudhry (1979).Biometrical Methods in Quantitative Genetics Analysis. 191-199. 2nd Ed. Kalyan: Publishers, New Delhi, India.

Tabassum, A.K. and B. Parasad (2017).Study of combining ability and nature of gene action for yield and its contributing traits in bread wheat (Triticumaestivum L. en Thell). Int. J. Curr. Microbial. App. Sci. 6(10): 3562-3573).

Titan, P.; V. Meglic and J. Iskra (2012).Combining ability and heterosis effect in hexaploid wheat group.Genetika 44: 595-609.

Verma, A. K.; S. R. Vishwakarma and P. K. Singh.(2007). Line $\mathrm{x}$ Tester analysis in barley (HordeumvulgareL.) across environments. Barley Genetics Newsletter 37: 29-33. 


\section{تقدير القدرة على التالف و الفعل الجينى باستخدام تكنيك السلالة x الكشاف فى قمح الخبز نسمه سالم حسين, محمود الزعبلاوى البلوى , احمد على الحصرى و صديق عبد العزيز صديق محيسن

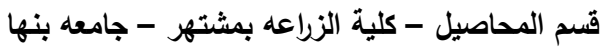

اقيمت التجربة لدراسة القدرة على التالف و الفعل الجينى لبعض صفات التبكير و المحصول و مكوناته و ايضا التفاعل مع معاملات الرى. اجرى بـ

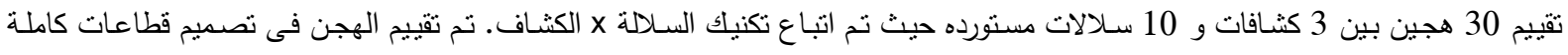
العشوائية و ذلك تحت معاملتين رى مختلفتين( رى عادى و جفاف) ـ كان منوسط التباين لمعاملتى الرى و التراكيب الوراثية و تجزئتها (سلالمة كثاف - سلالة x كثاف) معنوى لمعظم الصفات تحت الدراسة. كان تباين القدرة العامة و الخاصة على التالف معنوى فى جميع الصفات تحت الدراسـة و الجزء الغير مضيف يتحكم فى اظهار معظم الصفات. كان التفاعل بين معاملتى الرى و جميع مصـادر التباين معنوى فى معظم

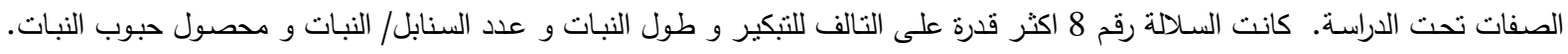
اظهرت الهجن T3 x L و T2 x L4T3 x L4, T2 x L2, T2 x L1 ,T1 x L3 قدرة خاصـة على التالف معنوية و موجبة لصفة محصول النبات البيولوجى و محصول حبوب النبات. 